Bài báo khoa học

\title{
Khả năng áp dụng các công thức bán thực nghiệm bùn cát đáy cho lưu vực sông có địa hình đáy dốc
}

\section{Đặng Trường An ${ }^{1 *}$}

${ }^{1}$ Trường ĐH Khoa học Tự Nhiên-Đại học Quốc gia Tp. HCM, 227 Nguyễn Văn Cừ, Quận 5, Tp.HCM; dtan@hcmus.edu.vn

*Tác giả liên hệ: dtan@hcmus.edu.vn; Tel.: +84-909719878

Ban Biên tập nhận bài: 12/3/2021; Ngày phản biện xong: 6/5/2021; Ngày đăng bài: 25/6/2021

Tóm tắt: Bùn cát đóng một vai trò quan trọng trong duy trì và ổn định hình thái sông. Trong đó, bùn cát đáy đóng góp đáng kể vào quá trình bồi-xói của sông. Các công thức tính lưu lượng bùn cát thường được sử dụng như một công cụ hữu dụng trong nghiên cứu về hình thái sông. Tuy nhiên, các công thức tính lưu lượng bùn cát thường xây dựng cho các mục đích áp dụng riêng, áp dụng giới hạn trong một số trường hợp cụ thể. Để triển khai, các công thức tính lưu lượng bùn cát của Park (2012), Cheng (2002), Meyer-Peter và Mueller (1950), Einstein-Brown (1950), Yalin (1963) và Van Rijn (2007) đã được áp dụng trong nghiên cứu này. Kết quả cho thấy, công thức Park tính lưu lượng bùn cát hợp lý đối với dữ liệu đã đo đạc. Sai số $R_{\text {mean, }}$ RMSE và MAPE lần lượt là 0,$75 ; 0,035$ và $10,5 \%$ trong khi các công thức khác các sai số này tương ứng dao động trong khoảng từ $0,31-0,71 ; 0,046-0,197$ và $16,8-$ 48,6\%. Công thức Park (2012) được xây dựng trên cơ sở phân cấp kích thước hạt, từ đó có thể khẳng định cách tiếp cận phân chia kích thước hạt trong tính toán lưu lượng bùn cát đáy đóng vai trò quan trọng chi phối kết quả.

Từ khóa: Úng suất cắt; Bùn cát đáy; Độ dốc; Kích thước hạt; Lưu lượng.

\section{Mở đầu}

Bùn cát đáy đóng một vai trò quan trọng đối với việc duy trì quá trình cân bằng và ổn định đáy của một con sông [1-3]. Do đó, việc tính toán, dự báo vận chuyển bùn cát là rất quan trọng trong nhiều dự án kỹ thuật liên quan đến sông ngòi $[2,4-6]$. Trong các nghiên cứu có liên quan biến đồi hình thái sông, các nhà chuyên môn thường tiến hành đo đạc các đặc trưng bùn cát ngoài hiện trường, tuy nhiên, các chuyến khảo sát đo đạc thu thập các mẫu bùn cát ngoài hiện trường thường rất khó khăn và tốn kém $[5,7-8]$. Một trong những giải pháp hiệu quả có thể giúp xác định lưu lượng bùn cát là áp dụng các công thức thực nghiệm và bán thực nghiệm để xác định lưu lượng bùn cát của một con sông $[3,5,9]$. Theo nghiên cứu [9], bùn cát tồn tại trong môi trường gần sát đáy sông theo cơ chế lăn, trượt, hoặc nhảy cóc thì được xem là bùn cát đáy. Mặc dù bùn cát đáy trong các con sông miền núi, sông có địa hình đáy dốc trong một số trường hợp chỉ chiếm tỉ lệ từ $5-25 \%$ so với bùn cát lơ lửng $[2$, 9-10], tuy nhiên chính bùn cát đáy là nhân tố quan trọng giúp duy trì thế cân bằng hay mất cân bằng địa hình đáy sông $[6,11]$. Chính vì vậy, kể từ những năm 1970 , nhiều nghiên cứu về chuyển vận bùn cát đáy đã được tiến hành ở các khu vực khác nhau trên thế giới [11-13].

Cụ thể, [12] đã tiến hành nghiên cứu xác định lưu lượng bùn cát đáy cho khu vực sông có vật liệu đáy thô. Nghiên cứu đã áp dụng 12 công thức tính lưu lượng bùn cát đáy để xác định lưu lượng bùn cát dựa trên tiếp cận chuyển vận bùn cát cân bằng cho 4 bộ dữ liệu đo đạc từ các con sông tự nhiên và 3 bộ dữ liệu đo được từ kênh thực nghiệm trong phòng thí Tap chi Khi tương Thủy văn 2021, 726, 25-35; doi:10.36335/VNJHM.2021(726).25-35 http://tapchikttv.vn/ 
nghiệm. Các kết quả nghiên cứu chỉ ra rằng, trong số các công thức đã áp dụng, không một công thức nào cho kết quả tính toán đáp ứng như mong đợi. Nghiên cứu đã rút ra nhận định rằng, thất bại trong nghiên cứu nguyên nhân chính đến từ việc các công thức tính lưu lượng bùn cát đã áp dụng được xây dựng dựa trên tiếp cận chuyển vận mà chưa xem xét bản chất vật lý của các hiện tượng xảy ra trong quá trình chuyển động của hạt. [13] đã áp dụng thử nghiệm một số công thức tính lưu lượng bùn cát đáy cho một đoạn sông có độ dốc đáy lớn Rio Cordo. Nghiên cứu đã chỉ ra rằng, công thức Bagnold [12] cho độ tin cậy cao thông qua hệ số tương quan giữa kết quả tính toán và thực đo. Năm 2002, [8] đã xây dựng một công thức bùn cát đáy dựa trên tiếp cận ứng suất cắt tới hạn để xác định lưu lượng bùn cát cho các con sông có vật liệu đáy thô. Qua quá trình nghiên cứu, tác giả đã báo cáo rằng, công thức cho kết quả tính toán phù hợp tốt trong môi trường có bùn cát chuyển động yếu đến chuyển động mạnh. [11] đã tiến hành đo đạc lưu lượng bùn cát tại Las Vegas Wash, một dòng chảy kênh Las Vegas của bang Nevada, Mỹ. Dữ liệu đo đạc này được sử dụng để nghiên cứu sự vận chuyển vật liệu đáy bằng một công thức bán thực nghiệm xác định tốc độ vận chuyển. Các kết quả tính toán chỉ ra rằng, chuyển động của bùn cát không chỉ phụ thuộc vào kích thước hạt mà còn phụ thuộc vào độ sâu dòng chảy. Công trình nghiên cứu này đã góp phần cải thiện hiểu biết của con người trong nghiên cứu các quá trình vận chuyển bùn cát tại các lưu vực sông có vật liệu đáy sông hòa trộn nhiều loại kích thước khác nhau. Năm 2012, [9] đã sử dụng 14 chuỗi dữ liệu đo đạc từ các con sông tự nhiên để ước lượng lưu lượng bùn cát đáy trong sông Node ở miền bắc Iran. Kết quả chỉ ra rằng, các công thức Van Rijn, MeyerPeter, Müller và Ackers, và White có thể áp dụng dự báo lưu lượng bùn cát cho khu vực sông Node. Một nghiên cứu được tiến hành bởi [1] đã đánh giá khả năng dự báo lưu lượng bùn cát đáy cho một số con sông thuộc Ấn Độ. Công thức Brown và Recking đã được áp dụng tính toán bùn cát đáy dựa trên dữ liệu đo đạc ngoài hiện trường. Kết quả cho thấy, công thức Recking tính lưu lượng tốt hơn công thức Brown.

Kết quả phân tích nghiên cứu cho thấy, hầu hết các công thức được xây dựng dựa trên đường kính hạt trung bình $\left(\mathrm{d}_{50}\right)$ hoặc sử dụng 3 loại kích thước hạt $\mathrm{d}_{35}, \mathrm{~d}_{50}$ và $\mathrm{d}_{85}$ [14-16] Theo [2, 9, 17-18], cách tiếp cận chỉ sử dụng kích thước hạt trung bình hay kết hợp 3 loại kích thước hạt cho kết quả tính toán chưa cao trong một số trường hợp lưu vực sông nghiên cứu có kích thước hạt từ cát mịn đến đá cuội. Do đó, mục tiêu của nghiên cứu là a) xác định công thức bùn cát đáy thích hợp ước tính lưu lượng bùn cát cho lưu vực sông Yangyang Namdaechon, Hàn Quốc và b) sử dụng công thức bùn cát được lựa chọn để xây dựng module mô phỏng diễn biến bồi-xói đáy sông cho nghiên cứu tiếp theo.

\section{Phương pháp nghiên cứu}

\subsection{Khu vục áp dụng nghiên cưu}

Khu vực nghiên cứu thuộc lưu vực sông Yangyang Namdaechon (Hình 1), phía đông bắc Hàn Quốc, có diện tích khoảng 128,68 $\mathrm{km}^{2}$ trải dài từ 37.6-38.5 vĩ độ Bắc và $128.3-$ 129.0 kinh độ Đông. Khu vực nghiên cứu bắt nguồn từ biên giới phía bắc Hàn Quốc, chảy xuống phía Nam với chiều dài sông chính xấp xỉ $100 \mathrm{~km}$. Địa hình khu vực nghiên cứu có độ dốc đáy sông dao động trong khoảng từ 1,5 đến $4,3 \%$. Khu vực nghiên cứu có khí hậu ôn hòa với mưa hàng năm trung bình khoảng $1400 \mathrm{~mm}$, trong đó mùa mưa chiếm đến $60 \%$ lượng mưa năm. Mưa trong bão có thể đạt đến 392 mm ghi nhận năm 2003, dẫn đến sạt lở đất và bùn cát trong các sông bao gồm cát, sỏi và đá cuội với đường kính hạt $\mathrm{d}_{50}$ dao động trong khoảng từ 2,35 đến $34.6 \mathrm{~mm}$ và thường đạt hàm lượng cực đại khi có bão xảy ra trong khu vực. Khi ấy, tốc độ dòng chảy có thể đạt $3,4 \mathrm{~m} / \mathrm{s}$ và lưu lượng dòng chảy khi ấy lên đến 190 $\mathrm{m}^{3} / \mathrm{s}[2]$. 


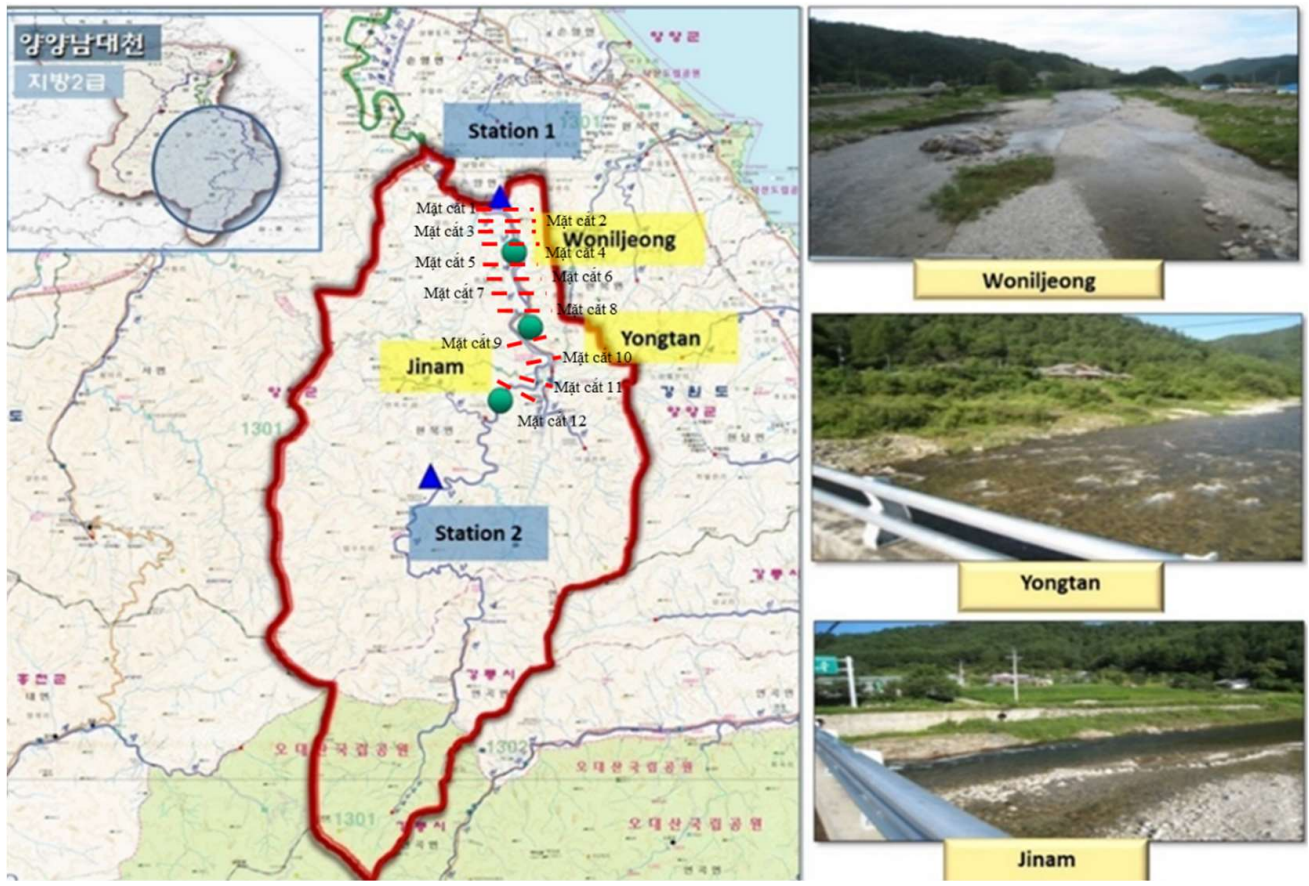

Hình 1. Bản đồ khu vực nghiên cứu.

\subsection{Phương pháp tiến hành}

Trong số các công thức tính lưu lượng bùn cát đáy hiện có, các nhà nghiên cứu thường tìm kiếm công thức đáp ứng một cách hiệu quả nhất đối với các vấn đề nghiên cứu của họ. Điều này không dễ dàng vì các công thức bùn cát khác nhau khi áp dụng cho một khu vực sẽ cho kết quả khác nhau đáng kể [13, 18-19]. Vì vậy, nghiên cứu này đã áp dụng thử nghiệm các công thức tính bùn cát đáy dựa trên khả năng ứng dụng của chúng cho các sông có vật liệu đáy đa dạng.

\subsubsection{Công thức của Park (2012)}

Năm 2012, Park và cộng sự đã xây dựng công thức bán thực nghiệm xác định lưu lượng bùn cát đáy dựa trên phân cấp kích thước hạt từ bộ số liệu khảo sát hiện trường các lưu vực sông miền núi ở Hàn Quốc [2]. Ý tưởng chính xây dựng công thức áp dụng cho các sông miền núi nơi mà vật liệu đáy không đồng nhất. Công thức được xây dựng như sau:

$$
\begin{gathered}
\mathrm{q}_{\mathrm{tbs}}=\sum_{\mathrm{i}=1}^{\mathrm{N}} \mathrm{q}_{\mathrm{sbi}} \\
q_{\mathrm{sb}}=\gamma \sqrt{g\left(\sigma_{s}-1\right) d_{m}^{3}} q_{\mathrm{sb}}^{*} \\
q_{\mathrm{sb}}^{*}=0.00157 \tau_{i}^{* 0.418}\left(\tau_{i}^{*}-\tau_{\mathrm{ci}}^{*}\right)^{0.307} \\
\tau_{\mathrm{ci}}^{*}=0.0308\left(\tau_{b} \frac{d_{i}}{d_{m}}\right)^{0.545} \\
\tau_{i}^{*}=\frac{\tau_{b}}{\gamma\left(\sigma_{s}-1\right) d_{i}}
\end{gathered}
$$

Trong đó $\mathrm{q}_{\mathrm{tbs}}$ trong công thức (1) là tổng lưu lượng bùn cát đáy $(\mathrm{kg} / \mathrm{s})$; $\mathrm{q}_{\mathrm{sb}}$ trong công thức trong công thức (2) là lưu lượng bùn cát ứng mỗi cấp kích thước hạt $\mathrm{d}_{\mathrm{i}}$ và $q_{\mathrm{sb}}^{*}$ là lưu lượng bùn cát đáy không thứ nguyên ứng với cấp hạt $\mathrm{i}$; $\mathrm{g}$ là gia tốc trọng trường $\left(\mathrm{m} / \mathrm{s}^{2}\right) ; \mathrm{d}_{\mathrm{m}}$ là kích thước hạt bùn cát trung bình chiếm $50 \%$ thành phần hạt mịn $(\mathrm{mm}) ; \tau_{\mathrm{ci}}{ }^{*}$ là ứng suất cắt Shields không thứ nguyên của chuyển động tới hạn; $\tau_{\mathrm{b}}$ là ứng suất cắt đáy không thứ nguyên; $\sigma_{\mathrm{b}}$ là tỷ số 
không thứ nguyên giữa $84 \%$ kích thước hạt bùn cát thô và $16 \%$ kích thước hạt bùn cát mịn và $\sigma_{\mathrm{s}}$ là tỷ số không thứ nguyên giữa trọng lượng riêng của bùn cát đáy và của nước.

$$
\tau_{b}=\gamma \mathrm{HS} ; \sigma_{b}=\left(\frac{d_{84}}{d_{16}}\right)^{\frac{1}{2}} ; \sigma_{s}=\frac{\gamma_{\mathrm{s}}}{\gamma}
$$

Trong đó $\mathrm{H}$ là độ sâu nước $(\mathrm{m}) ; \mathrm{d}_{\mathrm{m}}$ là kích thước hạt bùn cát đáy $(\mathrm{mm})$; $\mathrm{S}$ là độ dốc mặt nước; $\gamma_{\mathrm{s}}$ và $\gamma$ là trọng lượng riêng của hạt bùn cát đáy và của nước $\left(\mathrm{kg} / \mathrm{m}^{3}\right)$.

\subsubsection{Công thức của Cheng (2002)}

[8] đã giới thiệu công thức tính lưu lượng bùn cát đáy và công thức này sử dụng chỉ ứng suất cắt tương tự như đối với công thức [18]. Công thức được đánh giá phù hợp tốt với công thức tính bùn cát đáy [18] cho điều kiện chuyển vận bùn cát là yếu và ứng suất cắt nhỏ. Lưu lượng bùn cát đáy được xác định như sau:

$$
q_{b}=13 \tau^{*^{3} / 2} \exp \left(-\frac{0.05}{\tau^{* 3 / 2}}\right)
$$

Trong đó $\mathrm{q}_{\mathrm{b}}$ là lưu lượng bùn cát đáy $(\mathrm{kg} / \mathrm{s}), \tau^{*}$ là tham số ứng suất cắt không thứ nguyên Shields.

\subsubsection{Công thức của Meyer-Peter and Mueller (1948)}

Một trong những công thức được sử dụng rộng rãi trong các tính toán lưu lượng bùn cát đáy được thể hiện trong kết quả nghiên cứu [15]. Thêm vào đó, công thức cũng được xem là công cụ hiệu quả áp dụng tính lưu lượng bùn cát đáy cho các nghiên cứu cơ bản cũng như các ứng dụng kỹ thuật. Công thức được xây dựng như sau:

$$
\begin{gathered}
q^{*}=8\left[\frac{q_{w}^{\prime}}{q_{w}}\left(\frac{K_{b}}{K_{r}}\right)^{3 / 2} \tau^{*}-0.047\right]^{3 / 2} \\
q^{*}=\frac{q_{b}}{\sqrt{\operatorname{Rgd}_{m} d_{m}}} \\
\tau^{*}=\frac{\tau_{0}}{\rho \operatorname{Rgd}}=\frac{\mathrm{HS}}{\mathrm{Rd}_{\mathrm{m}}}
\end{gathered}
$$

Trong đó $\mathrm{q}_{\mathrm{b}}$ là lưu lượng bùn cát đáy $(\mathrm{kg} / \mathrm{s}), \mathrm{q}^{*}$ lưu lượng bùn cát không thứ nguyên; $\mathrm{g}$ là gia tốc trọng trường $\left(\mathrm{m} / \mathrm{s}^{2}\right) ; \mathrm{q}_{\mathrm{w}}$ là lưu lượng nước trên một đơn vị chiều rộng kênh $\left(\mathrm{m}^{3} / \mathrm{s} / \mathrm{m}\right) ; \mathrm{q}^{\prime}$ w là lưu lượng nước trên một đơn vị chiều rộng kênh sau khi hiệu chỉnh các hiệu ứng thành bên $\left(\mathrm{m}^{3} / \mathrm{s} / \mathrm{m}\right) ; \mathrm{K}_{\mathrm{b}}$ là hệ số nhám Manning-Strickler cho đáy kênh; $\mathrm{K}_{\mathrm{r}}$ là hệ số nhám ManningStrickler liên quan đến ma sát của đáy kênh; $\tau^{*}$ là ứng suất cắt không thứ nguyên Shields; $\tau_{0}$ là ứng suất cắt biên dưới điều kiện dòng chảy chuẩn và $\mathrm{d}_{\mathrm{m}}$ là đường kính hạt trung bình $(\mathrm{mm})$.

\subsubsection{Công thức của Einstein (1950)}

Công thức bùn cát đáy [20] được đặt tên theo tác giả. Công thức được xây dựng như sau:

$$
\begin{aligned}
& \mathrm{q}^{*}= \begin{cases}\frac{K \exp \left(-\frac{0.391}{\tau^{*}}\right)}{0.465} & \tau^{*}<0.182 \\
40 K \tau^{* 3} & \tau^{*} \geq 0.182\end{cases} \\
& K=\sqrt{\frac{2}{3}+\frac{36}{\mathrm{~d}^{* 3}}}-\sqrt{\frac{36}{\mathrm{~d}^{* 3}}}
\end{aligned}
$$

Trong đó $q^{*}$ là lưu lượng bùn cát đáy $(\mathrm{kg} / \mathrm{s})$; $\tau^{*}$ là ứng suất cắt không thứ nguyên Shields; $\mathrm{K}$ là hệ số thường được xác định dựa trên bảng tra và $\mathrm{d}^{*}$ là đường kính hạt bùn cát trung bình $(\mathrm{mm})$. 


\subsubsection{Công thức của Yalin (1963)}

Công thức bùn cát đáy [15] được xây dựng dựa trên tiếp cận ứng suất cắt. Công thức được miêu tả như sau:

$$
\mathrm{q}_{\mathrm{c}}=A\left(\frac{\rho_{s}}{\rho}-1\right) \rho^{\frac{1}{2}} \tau^{\frac{1}{2}} D_{s}
$$

Trong đó $\mathrm{q}_{\mathrm{c}}$ là lưu lượng hạt bùn cát $(\mathrm{kg} / \mathrm{s}) ; \rho_{\mathrm{s}}$ và $\rho_{\mathrm{r}}$ là mật độ bùn cát và nước $\left(\mathrm{kg} / \mathrm{m}^{3}\right)$; $\tau^{2}$ ứng suất cắt, $\mathrm{Ds}$ là đường kính hạt bùn cát trung bình $(\mathrm{mm}) ; \mathrm{g}$ là gia tốc trọng trường $\left(\mathrm{m} / \mathrm{s}^{2}\right)$ và $\mathrm{A}$ là hệ số được xác định bởi công thức (14). Trong khi tham số $\beta$ trong công thức (14) được xác định dựa trên công thức (15).

$$
\begin{array}{r}
A=0.635 \gamma\left[1-\frac{1}{\beta} \log (1+\beta]\right. \\
\beta=2.45 \frac{\sqrt{\tau_{i}^{*}}}{\left(\frac{\rho_{S}}{\rho}\right)^{0.4}}
\end{array}
$$

\subsubsection{Công thức của Rottner (1950)}

Rottner đã xây dựng công thức tính lưu lượng bùn cát đáy dựa trên các phân tích hồi quy [9]. Công thức được viết như sau:

$$
q_{b}=\gamma_{s}\left[(\gamma-1) \mathrm{gD}^{3}\right]^{\frac{1}{3}} \times\left\{\frac{V}{[(\gamma-1) \mathrm{gD}]^{\frac{1}{2}}}\left[0.667\left(\frac{d_{50}}{D}\right)^{\frac{2}{3}}+0.04\right]-0.778\left(\frac{d_{50}}{D}\right)^{\frac{2}{3}}\right\}^{3}
$$

Trong đó $\mathrm{q}_{\mathrm{q}}$ là lưu lương bùn cát đáy $(\mathrm{kg} / \mathrm{s}) ; \gamma_{\mathrm{s}}$ và $\gamma$ là trọng lượng riêng của hạt bùn cát đáy và của nước $\left(\mathrm{kg} / \mathrm{m}^{3}\right) ; \mathrm{g}$ là gia tốc trọng trường $\left(\mathrm{m} / \mathrm{s}^{2}\right) ; \mathrm{D}$ là độ sâu nước $(\mathrm{m}) ; \mathrm{V}$ vận tốc dòng chảy $\left(\mathrm{m} / \mathrm{s}^{2}\right) ; \mathrm{d}_{50}$ đường kính hạt bùn cát trung bình $(\mathrm{mm})$.

Các công thức sau đã được chọn để đánh giá khả năng tính lưu lượng bùn cát trong lưu vực sông Yangyang Namdaechon [2, 8-9, 13, 20]. Quy trình thực hiện nghiên cứu được trình bày ở hình 2 .

\subsection{Thu thập dĩ liệu}

Dữ liệu đo đạc thực địa tại 12 mặt cắt ngang của 3 đoạn sông Wonijeong, Yongtan và Jinam như minh họa ở Hình 1. Trong đó, các đoạn sông Wonijeong, Yongtan và Jinam cách lưu vực sông Yangyang Namdaechon lần lượt là $5 \mathrm{~km}, 10 \mathrm{~km}$ và $15 \mathrm{~km}$ về phía hạ lưu. Các dữ liệu đo đạc một số đặc trưng hình thái của các đoạn sông tại 12 mặt cắt ngang và các mẫu bùn cát đáy được thể hiện ở Bảng 1 .

Bảng 1. Các đặc trưng hình thái sông được khảo sát tại ba đoạn sông thuộc khu vực nghiên cứu.

\begin{tabular}{ccccccc}
\hline Vị trí & Mặt cắt & $\begin{array}{c}\text { Khoảng cách từ } \\
\text { cuối dòng sông } \\
(\mathrm{km})\end{array}$ & $\begin{array}{c}\text { Mực đáy } \\
(\mathrm{m})\end{array}$ & $\begin{array}{c}\text { Độ rộng } \\
\text { kênh }(\mathrm{m})\end{array}$ & $\begin{array}{c}\text { Độ dốc } \\
\text { kênh }(\mathrm{m} / \mathrm{m})\end{array}$ & $\begin{array}{c}\text { Đường kính hạt } \\
\text { trung bình }(\mathrm{mm})\end{array}$ \\
\hline \multirow{6}{*}{ Wonijeong } & Mặt cắt 1 & 0,0 & 5,2 & 49,3 & 0,0008 & 52,4 \\
& Mặt cắt 2 & 13,0 & 57,5 & 35,4 & 0,0130 & 51,7 \\
& Mặt cắt 3 & 14,0 & 65,0 & 44,7 & 0,0038 & 46,2 \\
& Mặt cắt 4 & 17,0 & 86,0 & 70,8 & 0,0065 & 56,4 \\
& Mặt cắt 5 & 19,0 & 104,6 & 83,4 & 0,0092 & 36,7 \\
Yongtan & Mặt cắt 6 & 22,0 & 128,6 & 66,0 & 0,0052 & 63,7 \\
& Mặt cắt 7 & 23,0 & 139,6 & 62,0 & 0,0133 & 73,5 \\
& Mặt cắt 8 & 24,0 & 148,8 & 69,5 & 0,0113 & 82,1 \\
& Mặt cắt 9 & 25,0 & 158,7 & 51,8 & 0,0154 & 16,8 \\
& Mặt cắt 10 & 26,0 & 171,5 & 52,5 & 0,0170 & 15,2 \\
& Mặt cắt 11 & 27,0 & 180,5 & 57,4 & 0,0091 & 59,3 \\
& Mặt cắt 12 & 27,2 & 183,4 & 63,8 & 0,0141 & 18,1 \\
\hline
\end{tabular}


Bên cạnh các đặc trưng hình thái và đường kính hạt bùn cát đáy được thu thập, độ sâu nước và lưu lượng dòng chảy cũng được thu thập (Hình 3 ) và đường cong phân bố phần trăm kích thước hạt tại 3 đoạn sông nghiên cứu cũng được phân tích và biểu thị như hình 4 .

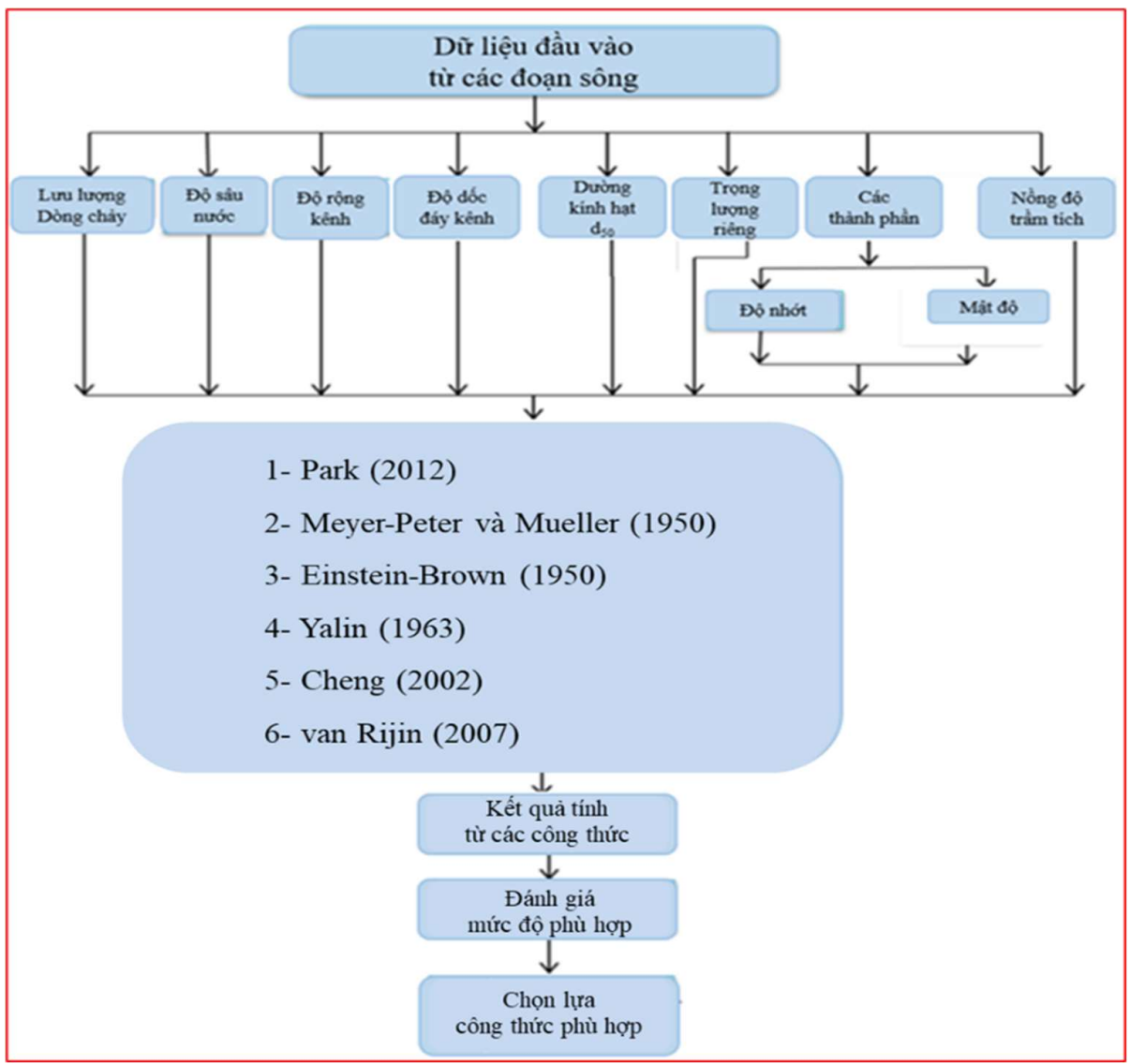

Hình 2. Biểu đồ minh họa quy trình tiến hành nghiên cứu.
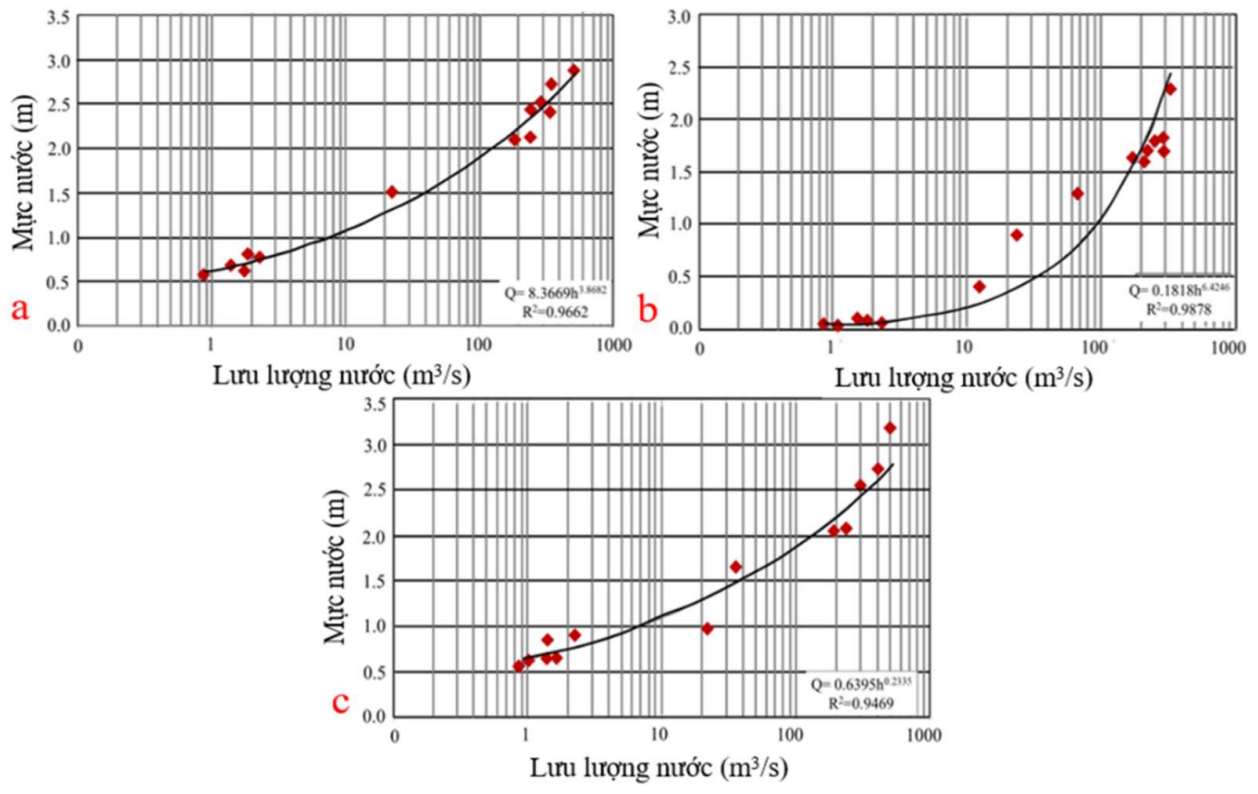

Hình 3. Quan hệ mực nước - lưu lượng nước tại a) mặt cắt số 3 b) mặt cắt số 7 và c) mặt cắt số 11 thuộc lưu vực sông nghiên cứu. 


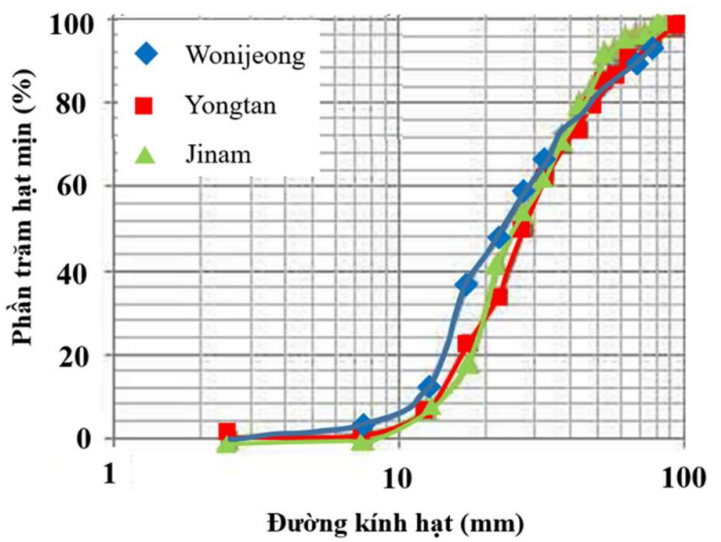

Hình 4. Đường cong phân bố kích thước hạt bùn cát đáy tại 3 mặt cắt (số 3,7 và số 11) thuộc khu vực nghiên cứu.

\subsection{Phân tích sai số}

Bốn tham số thống kê gồm tỷ lệ sai khác trung bình $\left(\mathrm{R}_{\text {mean }}\right)$, sai số toàn phương trung bình (RMSE) và phần trăm sai số tuyệt đối trung bình (MAPE) được sử dụng để xác định mức độ phù hợp của các công thức tính bùn cát và được thể hiện trong bảng 2 .

Bảng 2. Các chỉ dẫn thống kê phổ biến để đánh giá kết quả tính lưu lượng bùn cát.

\begin{tabular}{lr}
\hline \multicolumn{1}{c}{ Chỉ dẫn sai số } & Công thức tính \\
\hline $\begin{array}{l}\text { Tỷ lệ sai khác } \\
\text { trung bình }\end{array}$ & $\mathrm{R}_{\text {mean }}=\frac{\sum_{i=1}^{n} \frac{S_{i}}{O_{i}}}{n}$ \\
$\begin{array}{l}\text { Sai số } \\
\text { toàn phương } \\
\text { trung bình }\end{array}$ & $\mathrm{RMSE}=\sqrt{\frac{\sum_{\mathrm{i}=1}^{\mathrm{n}}\left(\mathrm{S}_{\mathrm{i}}-\mathrm{O}_{\mathrm{i}}\right)^{2}}{\mathrm{n}}}$ \\
$\begin{array}{l}\text { Phần trăm sai số } \\
\text { tuyệt đối trung bình }\end{array}$ & $\mathrm{MAPE}=\frac{\sum_{\mathrm{i}=1}^{n} \frac{A B S\left(S_{i}-\mathrm{O}_{i}\right)}{S_{i}}}{N}$ \\
\hline
\end{tabular}

Trong đó $\mathrm{S}_{\mathrm{i}}$ là dữ liệu bùn cát đáy tính toán; $\mathrm{O}_{\mathrm{i}}$ và $\mathrm{O}_{\mathrm{i}}$ lần lượt là dữ liệu bùn cát đáy đo đạc và dữ liệu bùn cát đáy trung bình.

\section{Kết quả và thảo luận}

Dựa trên các dữ liệu thủy động lực học, các đặc trưng hình thái và phân bố kích thước vật liệu bùn cát đáy đã thu thập được tại 3 khu vực sông thuộc đoạn sông nghiên cứu. Các kết quả tính toán lưu lượng bùn cát đáy từ 6 công thức được lựa chọn được thể hiện ở bảng 3 .

Bảng 3. Các kết quả tính toán lưu lượng bùn cát đáy $\left(\mathrm{q}_{\mathrm{sb}}\right) \mathrm{kg} / \mathrm{s} / \mathrm{m}$ từ các công thức đã áp dụng.

\begin{tabular}{ccccccc}
\hline Vị trí & $\begin{array}{c}\text { Park } \\
\mathbf{( 2 0 1 2 )}\end{array}$ & $\begin{array}{c}\text { Cheng } \\
\mathbf{( 2 0 0 2 )}\end{array}$ & $\begin{array}{c}\text { Meyer-Peter và } \\
\text { Mueller (1950) }\end{array}$ & $\begin{array}{c}\text { Einstein- } \\
\text { Brown (1950) }\end{array}$ & $\begin{array}{c}\text { Yalin } \\
\mathbf{( 1 9 6 3 )}\end{array}$ & $\begin{array}{c}\text { Van Rijn } \\
(\mathbf{2 0 0 7 )}\end{array}$ \\
\hline Mặt cắt 1 & 0,369 & 0,145 & 0,802 & 0,060 & 0,067 & 0,292 \\
Mặt cắt 2 & 0,359 & 0,678 & 0,125 & 1,015 & 1,046 & 0,535 \\
Mặt cắt 3 & 0,177 & 0,367 & 0,013 & 0,006 & 0,203 & 0,195 \\
Mặt cắt 4 & 0,530 & 0,077 & 0,038 & 1,027 & 1,490 & 0,975 \\
Mặt cắt 5 & 0,639 & 0,091 & 0,178 & 0,436 & 1,125 & 1,239 \\
Mặt cắt 6 & 0,391 & 0,052 & 0,094 & 0,012 & 0,692 & 0,416 \\
Mặt cắt 7 & 0,615 & 0,129 & 0,363 & 1,008 & 0,178 & 0,150 \\
Mặt cắt 8 & 0,739 & 0,064 & 0,034 & 0,368 & 0,674 & 0,483 \\
Mặt cắt 9 & 0,745 & 0,102 & 0,910 & 0,217 & 0,580 & 0,732 \\
\hline
\end{tabular}




\begin{tabular}{ccccccc}
\hline Vị trí & $\begin{array}{c}\text { Park } \\
\mathbf{( 2 0 1 2 )}\end{array}$ & $\begin{array}{c}\text { Cheng } \\
\mathbf{( 2 0 0 2 )}\end{array}$ & $\begin{array}{c}\text { Meyer-Peter và } \\
\text { Mueller (1950) }\end{array}$ & $\begin{array}{c}\text { Einstein- } \\
\text { Brown (1950) }\end{array}$ & $\begin{array}{c}\text { Yalin } \\
(\mathbf{1 9 6 3 )}\end{array}$ & $\begin{array}{c}\text { Van Rijn } \\
(\mathbf{2 0 0 7})\end{array}$ \\
\hline Mặt cắt 10 & 0,131 & 0,012 & 0,129 & 0,003 & 0,047 & 0,037 \\
Mặt cắt 11 & 2,548 & 0,501 & 0,017 & 2,183 & 0,037 & 0,015 \\
Mặt cắt 12 & 0,797 & 0,891 & 0,080 & 2,310 & 0,038 & 0,026 \\
qsb (tb) & $\mathbf{0 , 6 7 0}$ & $\mathbf{0 , 2 5 9}$ & $\mathbf{0 , 2 3 2}$ & $\mathbf{0 , 7 2 0}$ & $\mathbf{0 , 5 1 5}$ & $\mathbf{0 , 4 2 5}$ \\
qsb (max) & $\mathbf{2 , 5 4 8}$ & $\mathbf{0 , 8 9 1}$ & $\mathbf{0 , 9 1 0}$ & $\mathbf{2 , 3 1 0}$ & $\mathbf{1 , 4 9 0}$ & $\mathbf{1 , 2 3 9}$ \\
qsb (min) & $\mathbf{0 , 1 3 1}$ & $\mathbf{0 , 0 1 2}$ & $\mathbf{0 , 0 1 3}$ & $\mathbf{0 , 0 0 3}$ & $\mathbf{0 , 0 3 7}$ & $\mathbf{0 , 0 1 5}$ \\
\hline
\end{tabular}

Bảng 4 trình bày kết quả phân tích mức độ phù hợp dựa trên các chỉ dẫn sai số $\mathrm{R}_{\text {mean, }}$ RMSE và MAPE cho các công thức tính lưu lượng bùn cát đáy đã áp dụng cho lưu vực sông Yangyang Namdea. Từ bảng 4 ta thấy, công thức tính lưu lượng bùn cát đáy [2] ghi nhận sai số thống kê $\left(\mathrm{R}_{\text {mean }}=0,75, \mathrm{RMSE}=0,035\right.$ và $\left.\mathrm{MAPE}=10,5 \%\right)$ trong khi các công thức tính lưu lượng bùn cát khác có $R_{\text {mean, }}$ RMSE và MAPE dao động từ $0,31-0,63 ; 0,072-0,197$ và $17,9-48,6 \%$. Đối

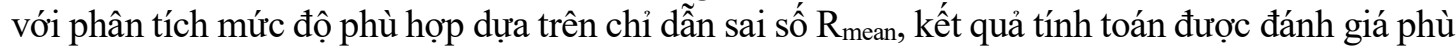
hợp tốt với dữ liệu đo đạc khi giá trị của chỉ số $\mathrm{R}_{\text {mean }}$ tiến đến 1 [19]. Theo đó, giá trị của chỉ số $\mathrm{R}_{\text {mean }}$ của công thức Park (2012) đạt $\mathrm{R}_{\text {mean }}=0,75$ và cao hơn so với các giá trị $\left(\mathrm{R}_{\text {mean }}=0,31\right.$ 0,63 ) của các công thức khác. Điều đó có nghĩa rằng, công thức [2] phù hợp hơn so với các công thức đã áp dụng khác (Hình 5).

Bảng 4. Kết quả thu được từ các sai số thống kê cho các công thức đã áp dụng.

\begin{tabular}{lccc}
\hline \multicolumn{1}{c}{ Công thức } & R $_{\text {mean }}$ & RMSE & MAPE \\
\hline Park (2012) & 0,75 & 0,035 & 10,5 \\
Cheng (2002) & 0,31 & 0,197 & 28,6 \\
Meyer-Peter và Mueller (1950) & 0,50 & 0,143 & 18,9 \\
Einstein-Brown (1950) & 0,63 & 0,164 & 48,6 \\
Yalin (1963) & 0,71 & 0,046 & 16,8 \\
Van Rijn (2007) & 0,63 & 0,072 & 21,9 \\
\hline
\end{tabular}

Đối với chỉ số RMSE, kết quả tính toán của một yếu tố được đánh giá có mức chính xác cao khi RMSE tiến gần đến giá trị 0 [19]. Bảng 4 cho thấy, chỉ số RMSE của công thức [2] cho giá trị nhỏ nhất $(\mathrm{RMSE}=0,035)$ so với các công thức khác $(\mathrm{RMSE}=0,049-0,197)$. Từ giá trị của chỉ số RMSE, có thể nhận định công thức tính bùn cát đáy [2] có sai số bé hơn so với sai số của các công thức tính khác. Một cách tương tự, kết quả phân tích phần trăm sai số tuyệt đối trung bình (MAPE) cũng cho thấy, so với các công thức khác, công thức tính lưu lượng bùn cát đáy của Park (2012) có sai số xấp xỉ 10,5\% trong khi các công thức khác có sai số dao động trong khoảng từ $14,8-48,6 \%$.
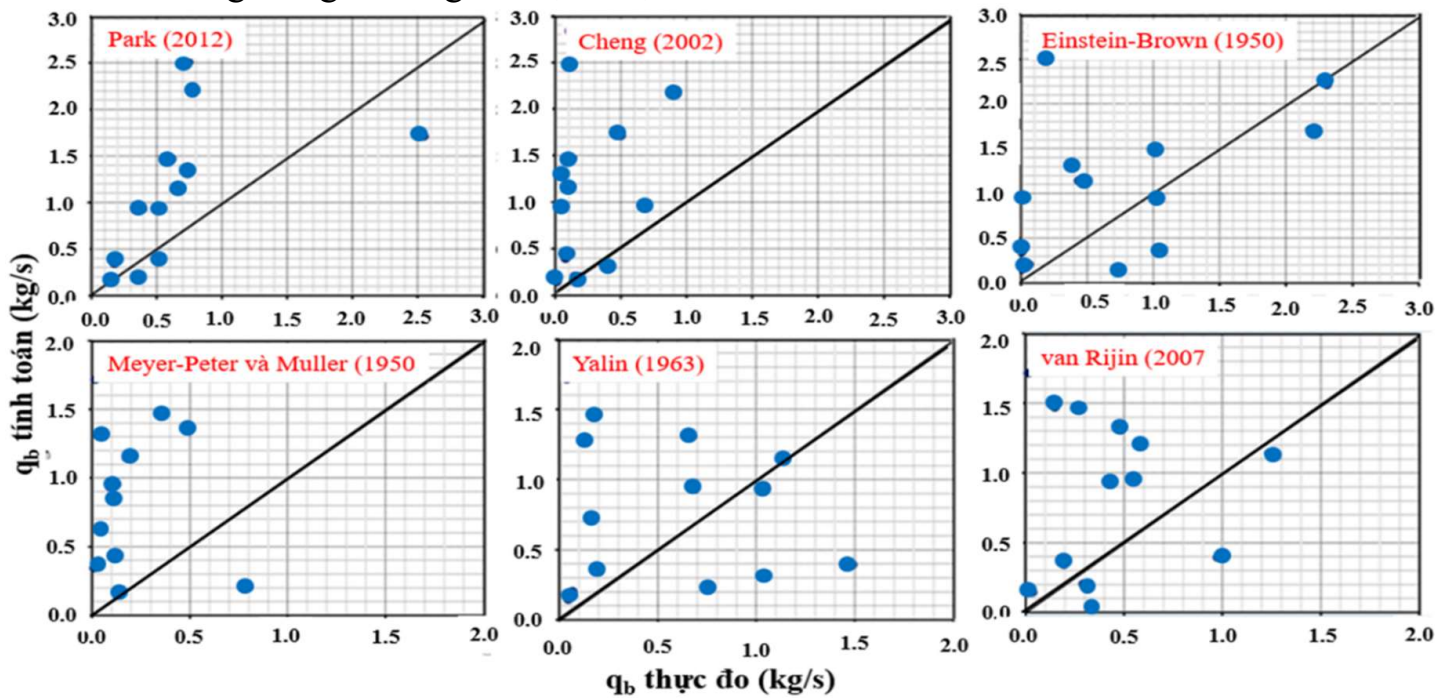

Hình 5. So sánh kết quả tính toán và đo đạc lưu lượng bùn cát đáy từ các công thức đã áp dụng. 
Mức độ phù hợp tốt của công thức Park (2012) có thể do điều kiện hình thái lưu vực sông nghiên cứu tương tự với các con sông được thu thập dữ liệu để xây dựng công thức. Công thức lưu lượng của Park (2012) được xây dựng trên cơ sở giả định rằng, bùn cát đáy của những con sông có địa hình đáy dốc với phân bố kích thước hạt rất đa dạng từ $1.25 \mathrm{~mm}$ đến $30.0 \mathrm{~mm}$. Thêm vào đó, trong 6 công thức tính lưu lượng bùn cát đáy đã áp dụng, hầu hết các công thức chỉ tính toán lưu lượng bùn cát dựa trên đường kính hạt trung bình $\left(\mathrm{d}_{50}\right)$ hay sử dụng 3 loại kích thước hạt đó là $\mathrm{d}_{35}, \mathrm{~d}_{50}$ và $\mathrm{d}_{85}$. Trong khi đó, chỉ công thức Park (2012) áp dụng phân cấp kích thước hạt từ $\mathrm{d}_{10}$ đến $\mathrm{d}_{100}$ trong tính toán lưu lượng. Đây có thể là một trong những khác biệt trong cách tiếp cận tính toán lưu lượng bùn cát trong công thức của Park (2012) và điều đó có thể góp phần tạo ra kết quả tính có mức độ phù hợp cao so với dữ liệu thực đo từ các con sông có phân cấp kích thước hạt đa dạng thuộc khu vực nghiên cứu.

\section{Kết luận}

Nghiên cứu đã áp dụng 6 công thức tính lưu lượng bùn cát đáy cho 3 bộ dữ liệu đã thu thập từ các đoạn sông khác nhau thuộc lưu vực sông Yangyang Namdeacheon, phía đông bắc Hàn Quốc. Các phân tích sai số cũng được áp dụng để đánh giá mức độ phù hợp của các công thức thông qua so sánh lưu lượng bùn cát tính toán từ các công thức và dữ liệu lưu lượng bùn cát thực đo.

Các kết quả thu được cung cấp cơ sở khoa học cho việc lựa chọn công thức bùn cát phù hợp xây dựng module mô phỏng thay đổi hình thái sông với độ dốc đáy lớn và vật liệu đáy đa dạng kích thước. Mức độ phù hợp của công thức tính thông qua các phân tích sai số cho thấy công thức của Park (2012) có mức độ phù hợp tốt hơn các công thức còn lại.

Tuy nhiên, mức độ phù hợp cao của một công thức bùn cát đáy được áp dụng cho một trường sông cụ thể không đồng nghĩa với công thức ấy có thể áp dụng tốt cho các lưu vực sông khác. Qua nghiên cứu, có thể nhận định độ chính xác của công thức tính bùn cát đáy ngoài phụ thuộc vào các yếu tố thủy động lực, đặc trưng hình thái sông thì phân bố kích thước hạt bùn cát đáy cũng đóng góp đáng kể vào kết quả tính của một công thức. Nhìn chung, rất cần có các đánh giá sơ bộ trước khi áp dụng công thức tính lưu lượng bùn cát cho một khu vực nghiên cứu cụ thể.

Đóng góp của tác giả: Tác giả đã tiến hành thực hiện tất cả các bước trong nghiên cứu này.

Lò̀i cảm ơn: Nghiên cứu này được thực hiện dưới sự hỗ trợ dữ liệu từ Phòng nghiên cứu động lực sông ngòi và Viện nghiên cứu giảm thiểu thiên tai Hàn Quốc. Tác giả xin chân thành cám ơn Giáo sư Park Sangdeog đã đóng góp ý kiến, nhận xét và hỗ trợ cơ sở dữ liệu để nghiên cứu được hoàn hành.

Lời cam đoan: Tác giả cam đoan bài báo này là công trình nghiên cứu của tác giả, chưa được công bố, không được sao chép từ những nghiên cứu trước đây; không có sự tranh chấp lợi ích trong nhóm tác giả.

\section{Tài liệu tham khảo}

1. Barry, J.J.; Bufngton, J.M.; King, J.G. Correction to a general power equation for predicting bed load transport rates in gravel bed rivers. Water Resour. Res. 2007, 43, W08702. http://dx.doi.org/10.1029/2007WR006103.

2. Park, S.D.; Lee, K.S.; Shin. S.S. Statistical soil erosion model for burnt mountain areas in Korea-RUSLE approach. J. Hydrol. Eng. 2012, 17, 292-304.

3. Schneider, J.M.; Rickenmann, D.; Turowski, B.; Schmid, B.; Kirchner, J.W. Bed load transport in a very steep mountain stream (Riedbach, Switzerland): 
Measurement and prediction. Water Resour. Res. 2016, 52, 9522-9541. http://dx.doi.org/10.1002/2016WR019308.

4. Bathurst, J.C.; Graf, W.H.; Cao, H.H. Bed load discharge equations for steep mountain rivers, sediment transport in gravel bed rivers. Wiley $U$. K. Chichester 1987, 453-477.

5. Haddadchi, A.; Omid, M.H.; Dehghani, A.A. Bedload equation analysis using bed load-material grain size. J. Hydrol. Hydromech. 2013, 61, 241-249.

6. Bathurst, J.C. Effect of coarse surface layer on bedload transport. J. Hydraul. Eng. 2007, 133, 1192-1205.

7. Bravo-Espinosa, M.; Osterkamp, W.R.; Lopes, V.L. Bedload transport in alluvial channels. J. Hydraul. Eng. 2003, 129, 783-795.

8. Cheng, N.S. Exponential formula for bed load transport. J. Hydraul. Eng. ASCE 2002, 128, 942-946.

9. Haddadchi, A.; Omid, M.H.; Dehghani, A.A. Assessment of bed-load predictors based on sampling in a gravel bed river. J. Hydrodyn. 2012, 24, 145-151.

10. An, D.T. Giáo trình vận chuyển trầm tích. NXB ĐH Quốc gia Tp.HCM. 2017

11. Duan, J.G.; Scott, S. Selective bed-load transport in Las Vegas Wash, a gravel-bed stream. J. Hydrol. 2007, 342, 320-330.

12. D'Agostino, V.; Lenzi, M. Bed-load transport in the instrumented catchment of the Rio Cordon Part II: Analysis of the bed-load rate. Catena, 1999, 36, 191-204.

13. Gomez, B.; Church, M. An assessment of bed loads sediment transport formulae for gravel bed rivers. Water Resour. Res. 1989, 25, 1161-1186.

14. Nasab, M.T.; Omid, M.H.; Farhoudi. J. Assessment of some bed load formulas based on sediment sampling. E-proceedings of the $36^{\text {th }}$ IAHR World Congress, The Hague, the Netherlands, 2015.

15. Nord, G.; Esteves, M. Evaluation of sediment transport formulae and detachment parameters in eroding rills using PSEM 2D and the Water Erosion Prediction Project (WEPP) database. Water Resour. Res. 2007, 43, W08420. http://dx.doi.org/10.1029/2006WR005444.

16. Reza, B.; Seyed, A.A.; Neyshabouri, S. Discussion of 'evaluation of bed load equations using field-measured bed load and bed material load by Sanjaykumar Madhusudan Yadav, Vipin Kumar Yadav, and Anurag Gilitwala. ISH J. Hydraul. Eng. 2019, 1-3. http://dx.doi.org/10.1080/09715010.2019.1630326.

17. Sun, Z.; Donahue, J. Statistically derived bedload formula for any fraction of nonuniform sediment. J. Hydraul. Eng. 2000, 126, 105-111.

18. Van Rijn, L.C. Unified view of sediment transport by currents and waves, I: Initiation of motion, bed roughness, and bed-load transport. J. Hydraul. Eng. 2007, 133, 649667.

19. Pourhosein, M.; Afzalimehr, H.; Singh, V.P.; Dehghani, A.A. Evaluation of bed load in a gravel-bed river. Int. J. Hydraul. Eng. 2015, 4, 70-79. http://dx.doi.org/10.5923/j.ijhe.20150403.03.

20. Song, T.Y.M.; Chin, C.O. Effect of bed load movement of flow friction factor. $J$. Hydr. Eng. ASCE 1998, 124, 165-175. 


\title{
Evaluation of the applicability of the semi-experimental bedload formulae for river basins with steep slope
}

\section{Dang Truong An ${ }^{1^{*}}$}

${ }^{1}$ University of Science -Vietnam National University-HCM City, 227 Nguyen Van Cu, District 5, HCMC; dtan@hcmus.edu.vn

\begin{abstract}
Sediments play an important role in maintaining and stabilizing river morphology. In which, bedload sediment contributes significantly to the aggradation and degradation processes. Bedload formulae are often used as a useful tool in studying the river morphological changes. However, bedload formulae are often developed for specific purposes and only apply the specific limitation cases. The study aims to a) select the appropriate bedload formulae for calculating sediment transport discharge in the Yangyang Namdaechon River basin, which located in the northeastern of South Korea and b) simulating the river morphological changes based on the selected bedload formulae in the next research. To conduct this work, six bedload formulae include Park (2012), Cheng (2002), Meyer-Peter and Mueller (1950), Einstein-Brown (1950), Yalin (1963) and Van Rijn (2007) have been applied. Results show that Park (2012) formula is good agreement to the measured data comparing other applied formulae. Specifically, the ratio of the calculated and measured bed load discharges $\left(\mathrm{R}_{\text {mean }}\right)$, Root Mean Square Error (RMSE) and Mean Absolute Percent Error (MAPE) of Park (2012) is 0.75, 0.035 and 10.5\% while other applied formulae are varying from $0.31-0.71 ; 0.046-0.197$ and $16.8-48.6 \%$, respectively. In general, Park (2012) formula is built based on the particle size fractions, which can confirm that the particle size fraction approach plays an important role for calculating bedload sediment discharge.
\end{abstract}

Keywords: Shear stress; Bedload sediment; Slope; Particle size; Discharge. 